\title{
Endovascular treatment of vertebrobasilar system aneurysms - long term results
}

\author{
Paweł Szmygin ${ }^{1}$, Maciej Szmygin ${ }^{2}$, Tomasz Roman ${ }^{2}$, Tomasz Jargiełło ${ }^{2}$ \\ ${ }^{1}$ Department of Neurosurgery, Medical University of Lublin, Lublin, Poland \\ ${ }^{2}$ Department of Interventional Radiology and Neuroradiology, Medical University of Lublin, Lublin, Poland
}

\begin{abstract}
Introduction. Microsurgical treatment of VBSA is complex and challenging due to the deep location of the vessels and the proximity of important structures. Therefore, minimally invasive endovascular techniques have been introduced to the treatment of VBSA. The aim of this study was to present long-term results and technical aspects of VBSA embolisation. In this manuscript, we describe our experience in the treatment of VBSA with long-term results. Furthermore, we discuss the advantages and disadvantages of this treatment modality, paying special attention to the technical aspects. We believe that this is an important contribution to this topic because the number of previous reports is limited. It adds to a population that can be compared to patients treated using different methods.

Materials and methods. We analysed the medical records of patients with aneurysms of vertebral and basilar arteries referred for endovascular treatment between 2015 and 2020.

Results. A total of 44 patients were selected for the study. From this group, 10 patients (22.7\%) were admitted with SAH caused by ruptured VBSA. The median age of the patients was 58 years (31-80), with $27(61.4 \%)$ females and 17 (38.6\%) males. The majority of the VBSAs (35, 79.5\%) were located on the basilar artery. Complete occlusion was achieved in 39 patients (88.6\%). Procedural complications occurred in three cases (6.8\%). In long-term follow-up, complete embolisation was achieved in $81.8 \%$ (36 of 44 patients). In 18.2\% (eight patients), recurrence of VBSA was observed. Two patients were qualified for recoiling. Clinical follow-up was uneventful in 37 (84\%) patients. Four patients (9\%) reported mild headaches. Three (7\%) patients required assistance for daily living.

Conclusions. Endovascular embolisation of VBSA is feasible and provides an excellent rate of complete occlusion and a high rate of favourable long-term outcomes, which support using this technique as a first-line treatment. A relatively high rate of recurrence remains the major drawback, especially in cases of wide-neck VBSA.
\end{abstract}

Key words: intracranial aneurysms, endovascular embolisation, long-term results, vertebrobasilar, complications

(Neurol Neurochir Pol 2021; 55 (6): 567-573)

\section{Introduction}

The vertebrobasilar system (VBS) consists of bilateral vertebral arteries and an unpaired basilar artery. It supplies the posterior portion of the brain including the occipital lobes, cerebellum, and brainstem [1]. Considering the area of supply, it is clear that any disruption to VBS circulation could have devastating clinical consequences. That is why vascular pathologies in this area require special attention.
Vertebrobasilar system aneurysms (VBSAs) accountforc.10-15\% of all cerebral aneurysms [2]. Similarly to aneurysms of anterior circulation, most VBSAs are asymptomatic and are nearly always identified incidentally during evaluation for other conditions [3]. However, cranial nerve deficits (e.g. isolated oculomotor nerve palsy) might be a classical clinical manifestation of VBSA [4]. The risk of rupture of aneurysms of posterior circulation is reported to be higher than that of anterior circulation $[5,6]$. Traditional neurosurgical treatment

Address for correspondence: Maciej Szmygin, Medical University of Lublin, Kazimierza Jaczewskiego Str. 8, 20-954, Lublin, Poland; e-mail: mszmygin@gmail.com Received: 1.09.2021 
of VBSA (i.e. clipping, by-pass surgery) is difficult due to the deep location of the vessels and the proximity of important structures, and therefore remains a significant challenge for neurosurgeons treating these aneurysms. Therefore, minimally invasive endovascular techniques have been introduced to the treatment of VBSA. Such treatment is much more feasible, enabling immediate post-operative angiographic control and carrying fewer of the risks which are inherent with open surgery [7]. Unfortunately, the authors of long-term studies have observed relatively high recurrence and retreatment rates after endovascular treatment [8].

The aim of this study was to summarise our six-year experience with endovascular treatment of VBSAs, with an emphasis on the technical aspects and procedural complications, and to present our short- and long-term results.

\section{Clinical rationale for study}

In this manuscript, we describe our experience in the treatment of VBSA with long-term results. Furthermore, we discuss the advantages and disadvantages of this treatment modality with special attention to the technical aspects. We believe that it adds to the limited number of reports on this topic, thus adding to a population that can be compared to patients treated using other means.

\section{Material and methods}

In this retrospective, single-centre study, we investigated the medical records of 44 patients with aneurysms of vertebral and basilar arteries who were referred for endovascular treatment to the Department of Interventional Radiology and Neuroradiology between 2015 and 2020. Clinical information including age, gender, risk factors and the pre-procedural clinical condition of all patients was collected. Ten patients experienced subarachnoid haemorrhage (SAH), seven were of Hunt-Hess grades I-II, and three were of grade III. Cerebral baseline imaging was performed with multi-slice computed tomography (CT) followed by either non-contrast CT or CT-angiogram or magnetic resonance angiography (MRA) to determine the diagnosis of VBSA.

Follow-up of the clinical outcome, ranging from 6 to 61 months (mean 28.3 months), was evaluated using the modified Glasgow Outcome Scale (mGOS) and was scored as follows: $1-$ minor deficits not hindering normal activity; 2 - independent with moderate disability; 3 - severely disabled and dependent on others for daily activities; $4-$ persistent vegetative state; 5 - deceased. Imaging follow-up data (MRA and/or DSA [Digital Subtraction Angiography]) ranged from 6 to 54 months (mean 23.6 months). This study was approved by our institutional review board (IRB).

All patients who were qualified for stent/flow diverter implantation or stent-assisted coiling received dual antiplatelet therapy combining aspirin $(150 \mathrm{mg})$ and clopidogrel $(75 \mathrm{mg}$ ) for five days before the procedure. In cases of ruptured aneurysms where no patient preparation was possible, patients received a double dose of aspirin and clopidogrel (i.e. $300 \mathrm{mg}$ of aspirin, $150 \mathrm{mg}$ of clopidogrel) immediately before the procedure and an additional intravenous heparin infusion for 24 hours after the embolisation (500 units/hour).

\section{Endovascular procedure}

All interventions were performed from femoral or radial access on a biplane angiographic unit with the patient under general anaesthesia and systemic heparinisation. Firstly, a 6F guiding catheter was advanced distally to the vertebral artery and initial rotational 3D angiography was performed. After evaluation of anatomical and haemodynamic conditions, embolisation was carried out by adopting one of the following methods: coiling, balloon/stent/pCONus assisted coiling, or flow diversion device placement. The aim of each endovascular intervention was complete angiographic elimination of the aneurysm, confirmed in control rotational angiography. Following the procedure which involved stent implantation, all patients received antiplatelet treatment: aspirin $(75 \mathrm{mg}$ daily) and clopidogrel (75 mg daily for a minimum of three months) after admission to the Department of Neurosurgery (up to 30 minutes after the procedure).

\section{Follow-up}

The clinical condition of the patient was evaluated 24 hours after the procedure and at discharge. Neurological status was measured on the basis of modified Glasgow Outcome Score (mGOS). Control MRA and/or DSA images were evaluated in all patients with a first control examination six months after the procedure.

\section{Results}

A total of 44 patients were selected for the study. Demographic data including age, medical history and pre-operational clinical condition is set out in Table 1 . The median age of patients was 58 years (31 to 80), with 27 (61.4\%) females and $17(38.6 \%)$ males. Five patients (11.4\%) reported a history of intracranial aneurysms and four $(9.1 \%)$ had a history of head injury. Mean blood pressure before the procedure was $143 / 87 \mathrm{mmHg}$, and mean heart rate was $83 \mathrm{bpm}$ (range 52 to 136). The most common risk factors included: hypertension (70\%), cardiovascular disease (previously known or diagnosed during hospitalisation) (45\%), smoking (36\%), diabetes mellitus (23\%), and hyperlipidemia (20\%).

Among patients with unruptured VBSA (34/44, 77\%), the most common symptoms included: vertigo and dizziness (33\%), headaches and nausea (27\%), and paraesthesia (16\%). Seven aneurysms (15.9\%) were found incidentally. In this group, four patients $(9.1 \%)$ had a positive Romberg's test. Ten patients (22.7\%) were admitted with SAH caused by ruptured VBSA.

All the patients underwent cerebral angiography. Three-dimentional reconstructions for measurement of aneurysmal 


\begin{tabular}{|c|c|}
\hline Patients - $44(n, \%)$ & $\begin{array}{cc}\text { Female } & \text { Male } \\
-27,61 \% & -17,39 \%\end{array}$ \\
\hline \multicolumn{2}{|l|}{ Demographic data } \\
\hline Mean age (years) (min-max) & $58(31-80)$ \\
\hline $\begin{array}{l}\text { History of intracranial aneurysms } \\
(n, \%)\end{array}$ & $5,11 \%$ \\
\hline History of head trauma $(n, \%)$ & $4,9 \%$ \\
\hline \multicolumn{2}{|l|}{ Clinical data } \\
\hline Mean blood pressure $(\mathrm{mmHg})$ & $143 / 87$ \\
\hline Mean heart rate (bpm) (min-max) & $83(52-157)$ \\
\hline \multicolumn{2}{|l|}{ Risk factors ( $n, \%)$} \\
\hline Hypertension & $31,70 \%$ \\
\hline Cardiovascular disease & $20,45 \%$ \\
\hline Smoking & $16,36 \%$ \\
\hline Diabetes mellitus & $10,23 \%$ \\
\hline Hyperlipidemia & $9,20 \%$ \\
\hline \multicolumn{2}{|l|}{ Unruptured VBSA (34, 77\%) } \\
\hline \multicolumn{2}{|l|}{ Aneurysm presentation $(\mathrm{n}, \%)$} \\
\hline Vertigo, dizziness & $19,43 \%$ \\
\hline Headaches, nausea & $16,36 \%$ \\
\hline Paraesthesia & $7,16 \%$ \\
\hline Short-term memory loss & $3,12 \%$ \\
\hline Tinnitus & $4,9 \%$ \\
\hline Loss of consciousness & $4,9 \%$ \\
\hline Neck/back pain & $2,5 \%$ \\
\hline Asymptomatic & $7,16 \%$ \\
\hline \multicolumn{2}{|l|}{ Ruptured VBSA (10, 23\%) } \\
\hline \multicolumn{2}{|l|}{ Hunt-Hess Grade $(n, \%)$} \\
\hline | or II & $7,70 \%$ \\
\hline III & $3,30 \%$ \\
\hline
\end{tabular}

sac, neck and diameter, as well as assessment of morphological features (direction of the aneurysmal dome projection, shape regularity) were performed in all cases (see Table 2). Most of the VBSAs $(35,79.5 \%)$ were located on the basilar artery. VBSA sizes were assigned to three categories: $<5 \mathrm{~mm}, 5-10 \mathrm{~mm}$, or $>10 \mathrm{~mm}$ in diameter. Ten aneurysms $(22.7 \%)$ were $<5 \mathrm{~mm}$, $29(65.9 \%)$ were $5-10 \mathrm{~mm}$, and five $(11.4 \%)$ were $>10 \mathrm{~mm}$. Mean neck width was $4.7 \mathrm{~mm}$ (range $1 \mathrm{~mm}$ to $10 \mathrm{~mm}$ ). Thirty-three $(75.0 \%)$ were classified as wide-necked aneurysms and $11(25.0 \%)$ as narrow-necked. The direction of the aneurysmal dome projection was classified into five types: anterior, posterior, superior, inferior, and lateral. More than half of VBSAs (23, 52.3\%) projected superiorly. Thirty-nine VBSAs $(88.6 \%)$ were defined as irregular (i.e. with one or more blebs).

Endovascular techniques included coiling (21, 47.7\%), balloon-assisted coiling (four, 9.1\%), stent-assisted coiling (12, 27.3\%), and stenting (seven, 15.9\%). Complete occlusion, defined as $>95 \%$ closure of the aneurysm in control on-table angiography, was achieved in 39 patients (88.6\%). Incomplete but successful occlusion (aneurysmal dome occlusions of
Table 2. Morphological features of VBSA

\begin{tabular}{|c|c|}
\hline \multicolumn{2}{|l|}{ Morphological features } \\
\hline \multicolumn{2}{|l|}{ Aneurysm location $\mathrm{n}, \%$} \\
\hline Basilar apex & $24,55 \%$ \\
\hline Basilar trunk & $11,25 \%$ \\
\hline Vertebrobasilar junction & $2,5 \%$ \\
\hline Vertebral artery & $5,10 \%$ \\
\hline Right & $2,5 \%$ \\
\hline \multicolumn{2}{|l|}{ Left } \\
\hline \multicolumn{2}{|c|}{ Aneurysm size (diameter) n, \% } \\
\hline$<5 \mathrm{~mm}$ & $10,23 \%$ \\
\hline $5-10 \mathrm{~mm}$ & $29,66 \%$ \\
\hline$>10 \mathrm{~mm}$ & $5,11 \%$ \\
\hline \multicolumn{2}{|l|}{ Neck width n, \% } \\
\hline Wide-necked aneurysms & $33,75 \%$ \\
\hline Narrow-necked aneurysms & $11,25 \%$ \\
\hline \multicolumn{2}{|c|}{ Direction of aneurysm projection $n, \%$} \\
\hline Superior & $23,52 \%$ \\
\hline Posterior & $8,18 \%$ \\
\hline Anterior & $5,11 \%$ \\
\hline Lateral & $5,11 \%$ \\
\hline Inferior & $3,8 \%$ \\
\hline \multicolumn{2}{|l|}{ Contour n, \% } \\
\hline Regular & $5,11 \%$ \\
\hline Irregular & $39,89 \%$ \\
\hline
\end{tabular}

90-95\%) was noted in three cases (6.8\%). Occlusions of $<90 \%$ were considered protective but incomplete and were observed in two patients $(4.5 \%)$. Procedural complications occurred in three cases (6.8\%). In all patients, coil protrusion into the parent artery was observed. Control angiography disclosed proper blood flow in all cases.

Imaging follow-up data was obtained for all patients and included DSA and/or MRA ranging from 6 to 54 months (mean 23.6) with first follow-up six months after the procedure. Complete embolisation was achieved in $81.8 \%$ (36 of 44) of patients. In $18.2 \%$ of patients (eight), recurrence of VBSA was observed. Recurrence was divided into three categories: 1) $<5 \%$ - clinically insignificant; 2) 5-10\% - requiring continued follow-up; 3 ) > 10\% - requiring thorough evaluation by a multidisciplinary board and potential retreatment. Four out of eight patients with recurrence (50.0\%) were classified as category 1 , and one (12.5\%) was classified as category 2 . Two patients $(25.0 \%$ of recurrent cases and $4.5 \%$ of all cases) were qualified for recoiling. All procedures were successful.

Forty $(90.9 \%)$ patients had uneventful postoperative courses and were classified as grade 1 or 2 in modified Glasgow Outcome Scale (independent in daily activities) at discharge. Four patients $(9.1 \%)$ required help in daily activities (mGOS-3) - due to neurological deficits related to ischaemia, which included visual impairment, paralysis and numbness. 
From the followed-up patients, $41(93.2 \%)$ had a good clinical outcome. They reported no long-term complications, except for mild headaches which could be managed with analgesic drugs in four cases. Three patients (6.8\%) needed assistance for daily living.

\section{Discussion}

Posterior circulation (PC) aneurysms, of which the majority are vertebrobasilar system aneurysms (VBSAs), account for 3.8-15\% of all intracranial aneurysms $[9,10]$. When compared to anterior circulation, endovascular treatment of PC aneurysms is burdened with higher rates of complication and reintervention [11]. However, several multicentre studies have demonstrated the superiority of endovascular techniques over surgical clipping in PC aneurysms treatment in terms of morbidity and neurological outcome, both in elective and emergency settings $[12,13]$.

The aim of this study was to summarise our six-year experience with endovascular treatment of VBSA with an emphasis on the technical aspects and procedural complications, and to present our short- and long-term results.

Our study included 44 patients with VBSA. The demographic characteristics of the patients, i.e. their mean age of 58 years (range 31 to 80 ) and the male-to-female ratio (the majority of the patients were male) was similar to other series [11, 14]. While most unruptured VBSAs remain asymptomatic and are diagnosed incidentally, symptomatic unruptured VBSAs are mainly diagnosed due to mass effect (large size) $[13,15]$.

Table 3. Procedural details and results

\begin{tabular}{lc} 
Procedural details & \\
Endovascular technique n, \% & \\
Coiling & $21,48 \%$ \\
Balloon-assisted coiling & $4,9 \%$ \\
Stent-assisted coiling & $12,27 \%$ \\
Stenting & $7,16 \%$ \\
Procedural outcome n, $\%$ & \\
Complete occlusion (> 95\%) & $39,89 \%$ \\
Successful occlusion (90-95\%) & $3,6 \%$ \\
Protective but incomplete occlusion (<90\%) & $2,5 \%$ \\
Coil protrusion & $3,7 \%$ \\
Results & \\
Imaging follow-up (6-54 months) n, $\%$ & \\
Complete embolisation & $36,82 \%$ \\
Recurrence & $3,18 \%$ \\
Insignificant (< 5\%) & $4,50 \%$ \\
Requiring observation (5-10\%) & $2,25 \%$ \\
Requiring reintervention (> 10\%) & $2,25 \%$ \\
Clinical follow-up n, $\%$ & \\
Complete recovery & \\
Mild headaches & \\
Needed assistance for daily living & \\
& \\
\hline
\end{tabular}

In our study, the most common symptoms included vertigo and dizziness, headaches and nausea, and paraesthesia. In a study which included a similar number of patients, Shi et al. reported neck pain, diplopia and ptosis, as well as hoarseness and dysphagia, as the most frequent initial symptoms among patients with an unruptured VBSA [16]. 23\% of our evaluated patients (10/44) presented with symptoms of subarachnoid haemorrhage (compared to the $34 \%$ reported by Shi et al.).

As far as the clinical results at admission were concerned, the majority of our patients were hypertensive and tachycardic (average blood pressure 143/87 $\mathrm{mmHg}$, average heart rate 83). In their recent paper, Nastasovic et al. concluded that increased blood pressure and HR are associated with unfavourable outcomes in patients treated due to ruptured intracranial aneurysms [17].

The most common location of the aneurysms in our group was the basilar apex (55\%). Even when performed by an expert, microsurgical clipping of basilar apex aneurysms remains one of the most challenging surgical procedures and is related to a relatively high complication rate (perforating vessel injury and ischaemia of midbrain or diencephalon) of $8-19 \%[18,19]$. On the other hand, endovascular treatment is associated with lower rates of long-term occlusions and a higher risk of retreatment $[20,21]$. According to the authors of a recent meta-analysis, as far as the endovascular technique is concerned, stent-assisted coiling provides a lower risk of recurrence compared to coiling but a higher risk of posttreatment haemorrhage [22]. Among 24 basilar apex aneurysms described in our study, eight (33\%) were treated with stent-assisted coiling, 12 (50\%) with coiling, three (13\%) with balloon-assisted coiling, and one (4\%) with stenting. Of this group, angiography immediately after the procedure

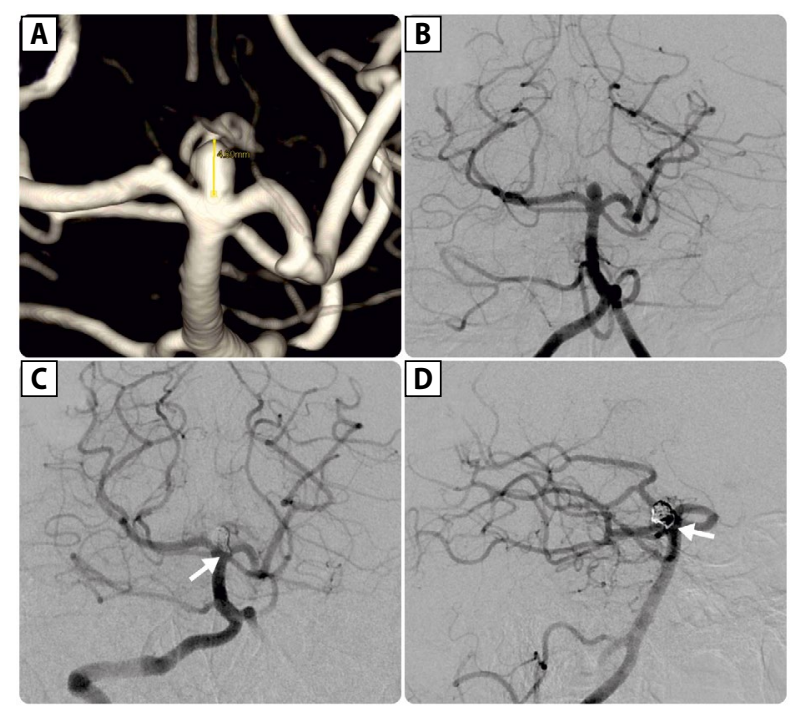

Figure 1. 3D CT-angio reconstruction and initial angiography in 49-year-old female patient with basilar apex aneurysm (A, B). Control angiography disclosed mild coil protrusion with no haemodynamic significance (white arrows) 


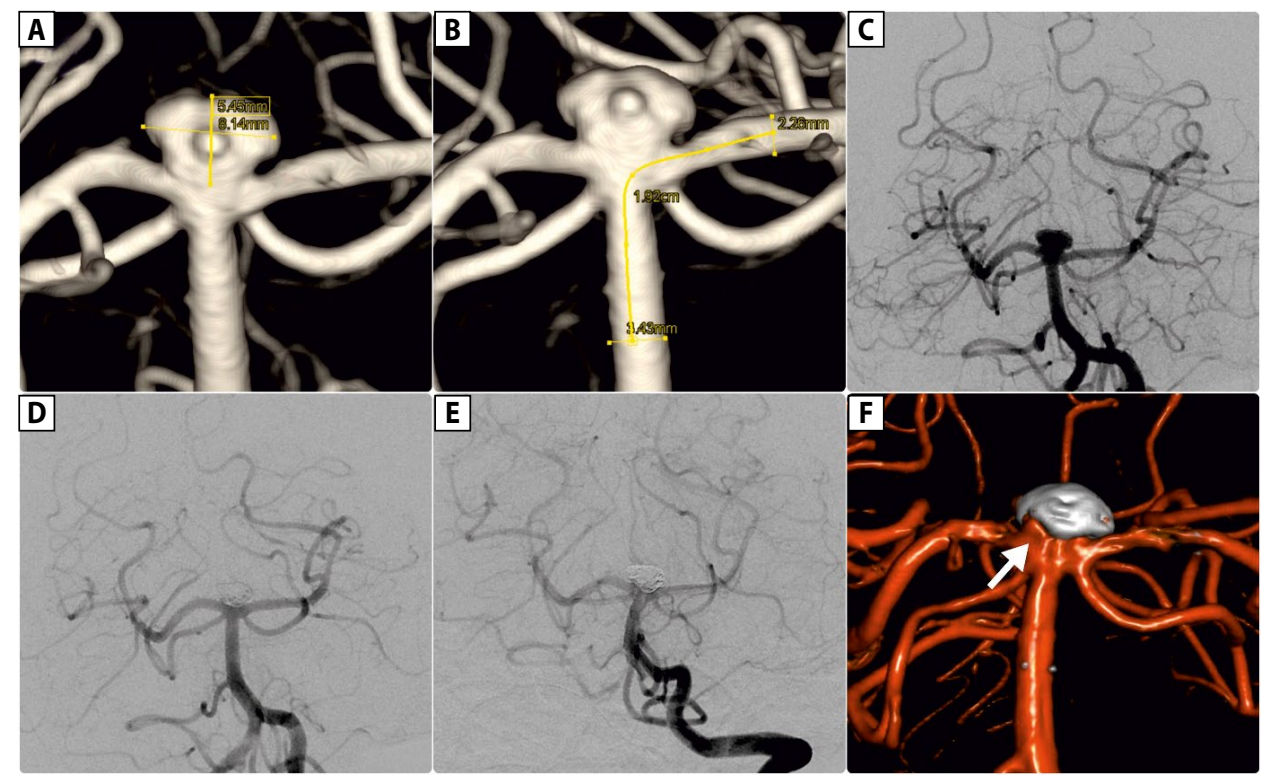

Figure 2. 3D CT-angio reconstruction and initial angiography of irregularly shaped basilar apex aneurysm in 63-year-old female patient (A-C). Due to its wide neck, stent-assisted coiling was performed. Final angiography showed proper stent and coils placement and no sign of aneurysmal filling (D). Control angiography performed six months after procedure disclosed proper stent patency and small filling of aneurysmal neck (E, F). Further control imaging examinations showed no haemodynamic changes

disclosed complete occlusion in $21(88 \%)$, successful occlusion in two (8\%), and protective occlusion in one (4\%). Long-term observation showed complete embolisation in $84 \%(20 / 24)$, insignificant recurrence in $8 \%(2 / 24)$, recurrence requiring observation in $4 \%(1 / 24)$, and significant recurrence requiring reintervention in $4 \%(1 / 24)$. This relatively high rate of recurrence might be attributable to the fact that basilar apex aneurysms generally have unfavourable anatomy (wider neck, larger domes, and incorporation into other branches) compared to other intracranial aneurysms [23]. The second most common location in our group was basilar artery trunk $-25 \%$. This rare type constitutes $2.1 \%$ of IC aneurysms [24]. Seven patients in our study (15\%) were treated for vertebral artery aneurysm, mainly on the right side. In high-volume studies, vertebral artery aneurysms have been found to constitute $2.8 \%$ of intracerebral aneurysms [25].

As far as morphological features of analysed VBSA are concerned, several aspects were evaluated. In terms of the size of the aneurysmal sac, 39 (89\%) aneurysms were $<1 \mathrm{~cm}$. Although the size of the aneurysm is a very important morphological aspect, dome-to-neck ratio remains the deciding factor in treatment planning. Wide-necked aneurysms require more advanced endovascular techniques than those required for narrow-necked aneurysms [26]. In our population, 75\% of aneurysms were classified as wide-necked (dome-to-neck ratio $<2$ ). In addition, the shape of the aneurysm is known to reflect the condition of the aneurysmal wall. In their study, Lindgren et al. presented the experience of a single centre over nearly 35 years, and concluded that an irregular or multilobular shape of an aneurysm is strongly associated with rupture, regardless of size, location or patient background [27]. In our study, 89\% of VBSAs (39/44) were classified as irregular. All patients who presented with symptoms of SAH and were diagnosed with a ruptured VBSA had irregularly-shaped aneurysms.

The complications of endovascular treatment of PC aneurysms range between $3 \%$ and $9.5 \%$ and include intraoperative rupture, catheter-induced vasospasm, thromboembolic event due to coil prolapse, and technical complications [11, 14, 28]. Compared to anterior circulation, a PC aneurysm rupture is less likely to cause medically refractory vasospasm (6.5\%), probably due to redundant collateral flow [29]. Mild coil protrusion is believed to be manageable with appropriate antiplatelet therapy [30]. However, moderate and severe protrusion might increase the risk of a thromboembolic event [31]. In our study, the observed rate of protrusion was 7\% $(3 / 44)$. All cases of protrusion were classified as mild and did not require any intervention.

A well-known concern of endovascular treatment is the long-term durability of the aneurysm occlusion. Established predictors of aneurysm recurrence after coiling are: treatment in the acute phase after rupture, aneurysm size, width of the neck of the aneurysm, and length of follow-up period [32, 33]. According to Lecler et al., an aneurysmal sac of $>10 \mathrm{~mm}$ multiplies the risk of recanalisation by more than four times [34]. Tomalski et al., who conducted a study on 107 patients with intracranial aneurysms treated endovascularly, confirmed these findings and concluded that large aneurysm volume may preclude coil stability and decrease coli density [35]. Tomalski et al. also highlighted the role of routine radiological check-up which allows assessment of the long-term results. In our series, overall recurrence rate in the long-term follow-up was $18 \%$, although only two patients $(5 \%)$ required reintervention. Both 
our recurrence and retreatment rates are comparable with the results reported by other authors $[7,8]$.

Our study has certain limitations. First and foremost, it is of retrospective design. Secondly, the cohort remains too small to perform subgroup analysis, and hence the validity of the data remains limited. Finally, the absence of a control arm comprising patients treated with clipping is a limitation. However, as the awareness of endovascular methods becomes more widespread, fewer patients are choosing microneurosurgery, and thus we cannot reliably compare and contrast the two treatment modalities.

\section{Clinical implications}

The results of our presented study of 44 patients with VBSA treated with endovascular embolisation demonstrate that this method is feasible and provides an excellent rate of complete occlusion and a high rate of favourable long-term outcomes, which support this technique as a first-line treatment. A relatively high rate of recurrence remains the major drawback, especially in cases of wide-neck VBSA.

Funding: This study was not supported by any funding. Conflicts of interest: The authors declare that they have no conflict of interest.

Ethical approval: All procedures performed in studies involving human participants were in accordance with the ethical standards of the institutional and/or national research committee and with the 1964 Helsinki Declaration and its later amendments or comparable ethical standards. The institutional review board (IRB) approved this study.

\section{References}

1. Piccinin MA, Munakomi S. Neuroanatomy, Vertebrobasilar System. StatPearls Publishing 2021.

2. Kim DJ, Heo Y, Byun J, et al. Role of microsurgery for treatment of posterior circulation aneurysms in the endovascular era. J Cerebrovasc Endovasc Neurosurg. 2020; 22(3): 141-155, doi: 10.7461/ jcen.2020.22.3.141, indexed in Pubmed: 32971573.

3. Keedy A. An overview of intracranial aneurysms. Mcgill J Med. 2006; 9(2): 141-146, indexed in Pubmed: 18523626.

4. Cianfoni A, Pravatà E, De Blasi R, et al. Clinical presentation of cerebral aneurysms. Eur J Radiol. 2013; 82(10): 1618-1622, doi: 10.1016/j.ejrad.2012.11.019, indexed in Pubmed: 23238357.

5. Rinkel GJ, Djibuti M, Algra A, et al. Prevalence and risk of rupture of intracranial aneurysms: a systematic review. Stroke. 1998; 29(1): 251-256, doi: 10.1161/01.str.29.1.251, indexed in Pubmed: 9445359.

6. Wermer MJH, van der Schaaf IC, Algra A, et al. Risk of rupture of unruptured intracranial aneurysms in relation to patient and aneurysm characteristics: an updated meta-analysis. Stroke. 2007; 38(4): 1404-1410, doi: 10.1161/01.STR.0000260955.51401.cd, indexed in Pubmed: 17332442.

7. Gruber DP, Zimmerman GA, Tomsick TA, et al. A comparison between endovascular and surgical management of basilar artery apex aneurysms. J Neurosurg. 1999; 90(5): 868-874, doi: 10.3171/ jns.1999.90.5.0868, indexed in Pubmed: 10223453.

8. Pandey AS, Koebbe C, Rosenwasser RH, et al. Endovascular coil embolization of ruptured and unruptured posterior circulation aneurysms: review of a 10-year experience. Neurosurgery. 2007; 60(4): 626-36; discussion 636, doi: 10.1227/01.NEU.0000255433.47044.8F, indexed in Pubmed: 17415199.

9. Zhao J, Wang S, Yang L, et al. Clinical experience of 153 patients with posterior circulation aneurysms. J Clin Neurosci. 2005; 12(1): 17-20, doi: 10.1016/j.jocn.2003.12.021, indexed in Pubmed: 15639405.

10. Bonneville F, Sourour N, Biondi A. Intracranial aneurysms: an overview. Neuroimaging Clin N Am. 2006; 16(3): 371-82, vii, doi: 10.1016/j. nic.2006.05.001, indexed in Pubmed: 16935705.

11. Lempert TE, Malek AM, Halbach VV, et al. Endovascular treatment of ruptured posterior circulation cerebral aneurysms. Clinical and angiographic outcomes. Stroke. 2000; 31(1): 100-110, doi: 10.1161/01. str.31.1.100, indexed in Pubmed: 10625723.

12. Molyneux A, Kerr R, Yu LM, et al. International subarachnoid aneurysm trial (ISAT) of neurosurgical clipping versus endovascular coiling in 2143 patients with ruptured intracranial aneurysms: a randomised comparison of effects on survival, dependency, seizures, rebleeding, subgroups, and aneurysm occlusion. The Lancet. 2005; 366(9488): 809-817, doi: 10.1016/s0140-6736(05)67214-5.

13. Wiebers D. Unruptured intracranial aneurysms: natural history, clinical outcome, and risks of surgical and endovascular treatment. The Lancet. 2003; 362(9378): 103-110, doi: 10.1016/s01406736(03)13860-3.

14. Lozier AP, Connolly ES, Lavine SD, et al. Guglielmi detachable coil embolization of posterior circulation aneurysms: a systematic review of the literature. Stroke. 2002; 33(10): 2509-2518, doi: 10.1161/01. str.0000031928.71695.a9, indexed in Pubmed: 12364746.

15. Toth G, Cerejo R. Intracranial aneurysms: Review of current science and management. Vasc Med. 2018; 23(3): 276-288, doi: 10.1177/1358863X18754693, indexed in Pubmed: 29848228.

16. Shi X, Qian H, Singh KC, et al. Surgical management of vertebral and basilar artery aneurysms: a single center experience in 41 patients. Acta Neurochir (Wien). 2013; 155(6): 1087-1093, doi: 10.1007/ s00701-013-1656-6, indexed in Pubmed: 23471600.

17. Nastasovic T, Milakovic B, Stosic M, et al. Predictors of unfavourable outcome in aneurysmal subarachnoid haemorrhage. Neurol Neurochir Pol. 2019; 53(6): 421-427, doi: 10.5603/PJNNS.a2019.0051, indexed in Pubmed: 31657445.

18. Samson D, Batjer HH, Kopitnik TA. Current results of the surgical management of aneurysms of the basilar apex. Neurosurgery. 1999; 44(4): 697-702; discussion 702, doi: 10.1097/00006123199904000-00001, indexed in Pubmed: 10201293.

19. Tjahjadi M, Kivelev J, Serrone JC, et al. Factors determining surgical approaches to basilar bifurcation aneurysms and its surgical outcomes. Neurosurgery. 2016; 78(2): 181-191, doi: 10.1227/ NEU.0000000000001021, indexed in Pubmed: 26361096.

20. Sekhar LN, Tariq F, Morton RP, et al. Basilar tip aneurysms: a microsurgical and endovascular contemporary series of 100 patients. Neurosurgery. 2013; 72(2): 284-98; discussion 298, doi: 10.1227/ NEU.0b013e3182797952, indexed in Pubmed: 23147787.

21. van Eijck M, Bechan RS, Sluzewski M, et al. Clinical and imaging follow-up of patients with coiled basilar tip aneurysms up to 20 years. AJNR Am J Neuroradiol. 2015; 36(11): 2108-2113, doi: 10.3174/ ajnr.A4410, indexed in Pubmed: 26185324. 
22. Dandurand C, Prakash S, Sepehry AA, et al. Basilar apex aneurysm: case series, systematic review, and meta-analysis. World Neurosurg. 2020; 138: e183-e190, doi: 10.1016/j.wneu.2020.02.064, indexed in Pubmed: 32084621.

23. $\mathrm{Ge} \mathrm{H}$, Lv X, Jin H, et al. The role of endovascular treatment in unruptured basilar tip aneurysms. Interv Neuroradiol. 2017; 23(1): 8-13, doi: 10.1177/1591019916665345, indexed in Pubmed: 27605544.

24. Saliou G, Sacho RH, Power S, et al. Natural history and management of basilar trunk artery aneurysms. Stroke. 2015; 46(4): 948-953, doi: 10.1161/STROKEAHA.114.006909, indexed in Pubmed: 25712945.

25. Ringer AJ, Lanzino G, Veznedaroglu E, et al. Does angiographic surveillance pose a risk in the management of coiled intracranial aneurysms? A multicenter study of 2243 patients. Neurosurgery. 2008; 63(5): 845-9; discussion 849, doi: 10.1227/01. NEU.0000333261.63818.9C, indexed in Pubmed: 19005373.

26. Hendricks BK, Yoon JS, Yaeger K, et al. Wide-neck aneurysms: systematic review of the neurosurgical literature with a focus on definition and clinical implications. J Neurosurg. 2019 [Epub ahead of print]: 1-7, doi: 10.3171/2019.3.JNS183160, indexed in Pubmed: 31200376.

27. Lindgren AE, Koivisto T, Björkman J, et al. Irregular shape of intracranial aneurysm indicates rupture risk irrespective of size in a population-based cohort. Stroke. 2016; 47(5): 1219-1226, doi: 10.1161/ STROKEAHA.115.012404, indexed in Pubmed: 27073241.

28. Ogilvy CS, Hoh BL, Singer RJ, et al. Clinical and radiographic outcome in the management of posterior circulation aneurysms by use of direct surgical or endovascular techniques. Neurosurgery. 2002; 51(1): 1421; discussion 21, doi: 10.1097/00006123-200207000-00003, indexed in Pubmed: 12182412.

29. Hirashima Y, Kurimoto M, Hori E, et al. Lower incidence of symptomatic vasospasm after subarachnoid hemorrhage owing to ruptured vertebrobasilar aneurysms. Neurosurgery. 2005; 57(6): 1110-6; discussion 1110, doi: 10.1227/01.neu.0000185632.69374.c9, indexed in Pubmed: 16331158.

30. Abdihalim M, Kim SH, Maud A, et al. Short- and intermediate-term angiographic and clinical outcomes of patients with various grades of coil protrusions following embolization of intracranial aneurysms. AJNR Am J Neuroradiol. 2011; 32(8): 1392-1398, doi: 10.3174/ ajnr.A2572, indexed in Pubmed: 21885722.

31. Ishihara $\mathrm{H}$, Ishihara $\mathrm{S}$, Niimi J, et al. Risk factors for coil protrusion into the parent artery and associated thrombo-embolic events following unruptured cerebral aneurysm embolization. Interv Neuroradiol. 2015; 21(2): 178-183, doi: 10.1177/1591019915582375, indexed in Pubmed: 25964443.

32. Raymond J, Guilbert F, Weill A, et al. Long-term angiographic recurrences after selective endovascular treatment of aneurysms with detachable coils. Stroke. 2003; 34(6): 1398-1403, doi: 10.1161/01. STR.0000073841.88563.E9, indexed in Pubmed: 12775880.

33. Tamatani S, Ito $\mathrm{Y}$, Abe $\mathrm{H}$, et al. Evaluation of the stability of intracranial aneurysms occluded with guglielmi detachable coils. Interv Neuroradiol. 2001; 7(Suppl 1): 143-148, doi: 10.1177/15910199010070S121, indexed in Pubmed: 20663392.

34. Lecler A, Raymond J, Rodriguez-Régent C, et al. Intracranial aneurysms: recurrences more than 10 years after endovascular treatment-a prospective cohort study, systematic review, and meta-analysis. Radiology. 2015; 277(1): 173-180, doi: 10.1148/radiol.2015142496, indexed in Pubmed: 26057784.

35. Tomalski W, Knap D, Żak A, et al. Recanalisation of cerebral artery aneurysms treated endovascularly - a midterm follow-up. Neurol Neurochir Pol. 2020; 54(6): 524-530, doi: 10.5603/PJNNS. a2020.0065, indexed in Pubmed: 32875548. 